\title{
AN EFFECTIVE NEUTROSOPHIC SET-BASED PREPROCESSING METHOD FOR FACE RECOGNITION
}

\author{
Mohammad Reza Faraji and Xiaojun Qi \\ Department of Computer Science, Utah State University, Logan, UT 84322-4205 \\ Email: Mohammadreza.Faraji@aggiemail.usu.edu, Xiaojun.Qi@usu.edu
}

\begin{abstract}
Face recognition (FR) is a challenging task in biometrics due to various illuminations, poses, and possible noises. In this paper, we propose to apply a novel neutrosophic set (NS)based preprocesssing method to simultaneously remove noise and enhance facial features in original face images. We then employ the Tan and Triggs (TT) discriminant method, which applies kernel fisher linear discriminant analysis (KFDA) on the linear ternary pattern (LTP) features, on the NS-based preprocessed images to further improve FR accuracy. Our experiments on two databases (ORL and FEI) show that the NSbased preprocessing method is more effective than other preprocessing methods to improve FR accuracy of discriminative methods. It can also be integrated with other preprocessing methods to further improve FR accuracy.
\end{abstract}

Index Terms - Face recognition, neutrosophic set, kernel fisher linear discriminant analysis, linear ternary pattern

\section{INTRODUCTION}

Face recognition (FR) is a good compromise between reliability and social acceptance and balances security and privacy well. It has been integrated in any application that requires security and access control [1]. However, illumination variations, pose changes, facial expressions, age variations, and occlusions significantly affect the performance of a FR system [1]. Thus, various preprocessing methods have been incorporated into the FR methods to achieve better FR accuracy.

Linear normalization (LN) and histogram equalization (HE) are two popular preprocessing methods for FR [2, 3]. Recently, Tan and Triggs [4] proposed a preprocessor (PP), which uses a chain of operations such as Gamma correction, Gaussian filtering, masking, and contrast equalization, to eliminate most of changing illumination effects. They also applied kernel fisher linear discriminant analysis (KFDA) on local ternary patterns (LTP) of the preprocessed image to achieve impressive FR accuracy. A comparative study of illumination preprocessing methods on FR [5] pointed out that HE, PP, gamma intensity correction (GIC), and logarithmic total variation (LTV) improve the FR accuracy.
On the other hand, the neutrosophic set (NS), as an extended version of the fuzzy set, has been recently used in image segmentation $[6,7]$. Researchers use the notations $T, I$, and $F$ to represent three neutrosophic components, e.g., truth, indeterminacy, and falsity degrees, respectively. So, a pixel in the NS domain is represented as $P\{T, I, F\}$, which means the pixel is $t \%$ true, $i \%$ indeterminate and $f \%$ false, where $t, i$, and $f$ vary in $T, I$, and $F$, respectively. Zhang et al. [6] proposed a $S$-function to map the intensity of each pixel to a value for $T$ in $[0,1]$, and then $F=1-T$. Sengur and Guo [7] used another transformation formula together with $\alpha$-mean and $\beta$-enhancement operations to map the intensity of each pixel to a value in $\{T, I, F\}$.

In this paper, we propose to apply a novel NS-based preprocesssing method for FR. Specifically, we first use entropies of the indeterminate subset and the candidate preprocessed face image to find appropriate ranges for three NS-based parameters. We then apply the NS operations on each image to simultaneously remove noise and enhance facial features. Finally, we uniquely combine this NS-based preprocessing method with the discriminative method to achieve better FR accuracy. To this end, we first apply the popular KFDA on preprocessed images to evaluate FR accuracy on two databases. We then use one state-of-the-art discriminant technique, the Tan and Triggs (TT) method, as the core to evaluate the effect of different preprocessing methods including the NS-based method, HE, LN, and PP on FR accuracy. Our extensive experiments on ORL and FEI databases demonstrate the following: 1) The proposed NSbased preprocessing method achieves the best FR accuracy when one preprocessing method is combined with the discriminant method. 2) The proposed NS-based method can be integrated with HE, LN, and PP to further improve FR accuracy when paired with the discriminant method. The rest of this paper is organized as follows: Section 2 presents our proposed NS operations for preprocessing face images. Section 3 shows the experimental results of applying the TT method on different preprocessed images obtained by one preprocessing method (NS, LN, HE, or PP) or combined prepocessing method (LN+NS, HE+NS, or PP+NS). Section 4 draws the conclusion and presents the directions of future work. 


\section{NEUTROSOPHIC SET-BASED PREPROCESSING}

NS is a new branch of philosophy to study the origin, nature, and scope of neutralities, as well as their interactions with different ideational spectra [8]. Each event has three degrees including truth, falsity and indeterminacy. We propose a novel preprocessing step for face images using NS-based operations. This step effectively removes noise and enhances facial features at the same time. Following NS-based segmentation techniques $[6,7]$, we transform the original image into the NS domain, which maps the gray-level intensity of each pixel to a value in $\{T, I, F\}$. We then apply the $\alpha$-mean and $\beta$-enhancement operations on $\{T, I, F\}$ to make the distribution of the elements in $I$ more uniform and the elements in $T$ and $F$ more distinct. Finally, we use a $\gamma$-decomposition operation to decompose $T$ into the final preprocessed image. We use the entropy to empirically determine $\alpha, \beta$ and $\gamma$ values to achieve good preprocessing results for FR.

2.1. $\alpha$-mean, $\beta$-enhancement, and $\gamma$-decomposition operations

We transform a pixel $P(i, j)$ with the intensity value of $g(i, j)$ in the image domain into the NS domain, $P_{N S}(i, j)=$ $\{T(i, j), I(i, j), F(i, j)\}$. These values are defined as follow:

$$
\begin{gathered}
T(i, j)=\frac{\bar{g}(i, j)-\bar{g}_{\min }}{\bar{g}_{\max }-\bar{g}_{\min }} \\
I(i, j)=\frac{\delta(i, j)-\delta_{\min }}{\delta_{\max }-\delta_{\min }} \\
F(i, j)=1-T(i, j)
\end{gathered}
$$

where $g(i, j)$ is the pixel intensity at position $(i, j), \bar{g}(i, j)$ is the local mean value of $g(i, j), \delta(i, j)=a b s(g(i, j)-\bar{g}(i, j))$, $\bar{g}_{\min }$ and $\bar{g}_{\text {max }}$ are respectively the minimum and the maximum of local mean values at all positions in an image, and $\delta_{\min }$ and $\delta_{\max }$ are respectively the minimum and the maximum of $\delta$ at all positions in an image. Here, we use the $3 \times 3$ square window to compute the local mean values.

The value of $I(i, j)$ measures the indeterminacy of element $P_{N S}(i, j)$. We first employ an $\alpha$-mean operation to increase the entropy of the indeterminate subset $I$ and make the distribution of the elements in $I$ more uniform. To this end, we make $T$ and $F$ correlate with $I$. In other words, the changes in $T$ and $F$ influence both the distribution of the elements and the entropy of $I$. This operation, smooths certain values in $T$ and $F$ based on the choice of $\alpha$. In addition, it increases the entropy of $I$. It is defined as follows:

$$
\begin{gathered}
\bar{P}_{N S}(\alpha)=P(\bar{T}(\alpha), \bar{I}(\alpha), \bar{F}(\alpha)) \\
\bar{T}(\alpha)=\left\{\begin{array}{cc}
T & I<\alpha \\
\bar{T}_{\alpha} & I \geq \alpha
\end{array}\right. \\
\bar{I}_{\alpha}(i, j)=\frac{\bar{\delta}_{T}(i, j)-\bar{\delta}_{\text {Tmin }}}{\bar{\delta}_{\text {Tmax }}-\bar{\delta}_{\text {Tmin }}} \\
\bar{F}(\alpha)=\left\{\begin{array}{cc}
F & I<\alpha \\
\bar{F}_{\alpha} & I \geq \alpha
\end{array}\right.
\end{gathered}
$$

where $\bar{T}_{\alpha}$ is the local mean value of $T, \bar{F}_{\alpha}$ is the local mean value of $F, \bar{\delta}_{T}(i, j)=\operatorname{abs}(\bar{T}(i, j)-\overline{\bar{T}}(i, j)), \bar{\delta}_{T \text { min }}$ and $\bar{\delta}_{\text {Tmax }}$ are respectively the minimum and the maximum of $\bar{\delta}_{T}$ at all positions in an image.

Next, we employ a $\beta$-enhancement operation to make the elements in $T$ and $F$ more distinct. It is defined as follows:

$$
\begin{gathered}
P_{N S}^{\prime}(\beta)=P\left(T^{\prime}(\beta), I^{\prime}(\beta), F^{\prime}(\beta)\right) \\
T^{\prime}(\beta)=\left\{\begin{array}{cc}
T & I<\beta \\
T_{\beta}^{\prime} & I \geq \beta
\end{array}\right. \\
T_{\beta}^{\prime}(i, j)=\left\{\begin{array}{cc}
2 T^{2}(i, j) & T(i, j) \leq 0.5 \\
1-2(1-T(i, j))^{2} & T(i, j)>0.5
\end{array}\right. \\
I_{\beta}^{\prime}(i, j)=\frac{\delta_{T}^{\prime}(i, j)-\delta_{\text {Tmin }}^{\prime}}{\delta_{\text {Tmax }}^{\prime}-\delta_{\text {Tmin }}^{\prime}} \\
F_{\beta}^{\prime}(i, j)=\left\{\begin{array}{cc}
F & I<\beta \\
F_{\beta}^{\prime} & I \geq \beta \\
2 F^{2}(i, j) & F(i, j) \leq 0.5 \\
1-2(1-F(i, j))^{2} & F(i, j)>0.5
\end{array}\right.
\end{gathered}
$$

where $\delta_{T}^{\prime}(i, j)=\operatorname{abs}\left(T^{\prime}(i, j)-\bar{T}^{\prime}(i, j)\right)$, and $\delta_{T \min }^{\prime}$ and $\delta_{\text {Tmax }}^{\prime}$ are respectively the minimum and the maximum of $\delta_{T}^{\prime}$ at all positions in an image.

Finally, we employ the $\gamma$-decomposition operation to decompose $T$ into the final preprocessed image $X$. This operation considers the effect of the indeterminancy $I$ to recompute the value for the true subset $T$. It is defined as follows:

$$
X(i, j)=\left\{\begin{array}{cc}
T(i, j) & I(i, j) \leq \gamma \\
\bar{T}_{\gamma}(i, j) & I(i, j)>\gamma
\end{array}\right.
$$

where $\bar{T}_{\gamma}$ is the local mean value of the $T$ set.

\subsection{Determination of $\alpha, \beta$, and $\gamma$ values}

We use the entropy of the indeterminate subset $I$ to find the range of $\alpha$ and $\beta$, and use the entropy of the candidate preprocessed image $X$ to find the range of $\gamma$. The entropy is chosen because its value can be interpreted as the quantity of information, i.e., the greater the entropy is, the more information the system includes [6]. The entropy of $I$ is computed by:

$$
E n_{I}=-\sum_{i=\min \{I\}}^{\max \{I\}} p_{i} \log \left(p_{i}\right) ; \quad p_{i}=N_{i} / N .
$$

where $N_{i}$ is the frequency of the rounded integer value $i$ in $I$ and $N=\sum_{i=0}^{L-1} N_{i}$. We can similarly compute the entropy of $X$, i.e., $E n_{X}$.

To find a suitable range for $\alpha, \beta$, and $\gamma$, for each combined $\alpha$ and $\beta$ pair ranging from 0 to 1 with the stepsize of 0.05 , we perform the $\alpha$-mean and $\beta$-enhancement operations and find the $(\alpha, \beta)$ pair with the highest entropy. Based on the results from the optimal $(\alpha, \beta)$ pair, for each $\gamma$ value ranging from 0 to 1 with the stepsize of 0.01 , we perform the $\gamma$ decomposition and find the $\gamma$ with the highest entropy. Fig. 1(a) shows $E n_{I}$ with regard to all experimented $\alpha$ and $\beta$ values for a face image. Fig. 1(b) shows $E n_{X}$ with regard to all experimented $\gamma$ values for the same image. $E n_{I}$ obtained its highest value when $\alpha$ and $\beta$ are close to zero and almost less 


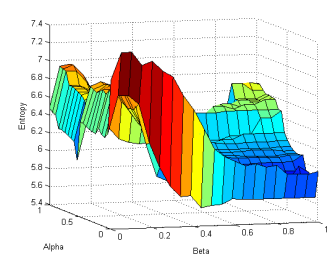

(a) $\left(E n_{I}, \alpha, \beta\right)$

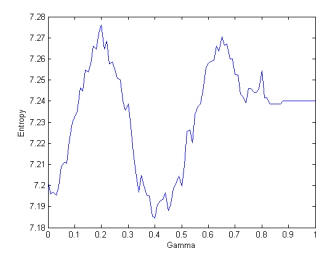

(b) $\left(E n_{X}, \gamma\right)$
Fig. 1. An entropy plot for a face image from ORL database. (Plots for other faces are similar)

than 0.25 , for almost $90 \%$ of face images from two databases. Similarly, $E n_{X}$ obtained its highest value when $\gamma$ value is close to zero but greater than both $\alpha$ and $\beta$ values. So, we consider $\alpha=\beta$ and $\gamma=\alpha+\beta$ in our system. The $\alpha$ value for each face is further computed by a linear scaling technique as:

$$
\alpha=\alpha_{\min }+\frac{\left(\alpha_{\max }-\alpha_{\min }\right)\left(E n_{I}-E n_{\min }\right)}{\left(E n_{\max }-E n_{\min }\right)}
$$

where $E n_{\text {max }}=-\log _{2} \frac{1}{h w}, h$ and $w$ are the height and width of an image, $E n_{\min }=0, \alpha_{\min }=0$ and $\alpha_{\max }=0.25$.

\subsection{Neutrosophic set-based preprocessing step}

We now use the $\alpha$ 's maximum value to quickly compute the optimal $\alpha$ value for each face image using Eq. (16). The algorithmic view of the proposed NS-based preprocessing step is summarized as follows:

1. Transform the original face image into the NS domain, $P_{N S}(i, j)=\{T(i, j), I(i, j), F(i, j)\}$, using Eqs 1-3.

2. Compute the entropy $E n_{I}\{$ old $\}$ of the subset $I$.

3. Compute $\alpha$ value using Eq. (16).

4. Assign $\alpha$ to $\beta$ and assign $\alpha+\beta$ to $\gamma$.

5. Sequentially perform the $\alpha$-mean and the $\beta$-enhancement operations on $P_{N S}(i, j)$.

6. Compute the entropy $E n_{I}\{$ new $\}$ of $I$.

7. If $\frac{\left(E n_{I}\{n e w\}-E n_{I}\{\text { old }\}\right)}{E n_{I}\{\text { old }\}}<\delta$, go to step 8, else assign $E n_{I}\{$ new $\}$ to $E n_{I}\{$ old $\}$ and go to step 5 .

8. Perform the $\gamma$-decomposition operation to compute $X$.

Our experiments on all face images show the convergence condidtion in step 7 is satisified after 3-4 iterations in most cases. As a result, this preprocessing step is fast after the offline process to learn the ranges for $\alpha, \beta$, and $\gamma$ values.

\section{EXPERIMENTAL RESULTS}

To evaluate the effectiveness of the proposed NS-based preprocessing method, we compare FR accuracy on two face databases (ORL and FEI) by combining different preprocessing methods with the discriminative method. To this end, we first apply the KFDA on the preprocessed images to evaluate its FR accuracy compared with FR accuaracy obtained by KFDA alone. We then use one state-of-the-art discriminant technique, the TT method, as the core to evaluate the effect of different preprocessing methods including NS, HE, $\mathrm{LN}, \mathrm{PP}, \mathrm{HE}+\mathrm{NS}, \mathrm{LN}+\mathrm{NS}$, and PP+NS on FR accuracy. For

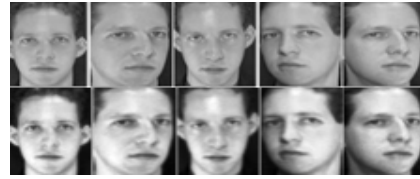

(a) ORL

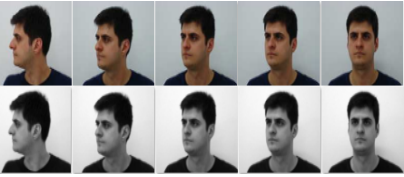

(b) FEI
Fig. 2. Sample images for one person and their preprocessed images from ORL and FEI databases

the kernels in KFDA and TT, we use the Gaussian kernel $\exp ^{\left(-\operatorname{dist}(X, Y) / 2 \sigma^{2}\right)}$, where $X$ and $Y$ are vectors in the input space, $\operatorname{dist}(X, Y)$ is the $\chi^{2}$ histogram distance, and $\sigma=10^{5}$ [4].

We use ORL and FEI databases, which have at least 10 images per subject, to evaluate the FR performance of our preprocessing method since ORL database is one of the most popular face databases and FEI database is a color face database. We randomly select 4,5 , and 6 images per person for training and the remaining images for testing. To report a more accurate performance of each method under each training, we average FR rates over 20 runs with each run being performed on a random partition of the databases.

\subsection{Recognition results on ORL database}

The ORL database contains 400 grayscale images $(92 \times 112$ pixels) of 40 individuals. They are taken at different times and slightly varying lighting conditions. They also have different facial expressions (open/closed eyes, smiling/non-smiling) and facial details (glasses/no-glasses). Fig. 2(a) shows a sample individual from this database and the preprocessed images at the top and bottom rows, respectively.

To speed up the processing time, we normalize all ORL face images to the size of $23 \times 28$ pixels. Table 1 shows the average FR rates obtained by combining different preprocessing methods and the discriminant method (e.g., KFDA or TT method) using a different number of training images. This table clearly shows that our NS-based preprocessing followed by KFDA improves KFDA by $0.93 \%, 0.89 \%$, and $0.57 \%$ in terms of FR accuracy using 4, 5, and 6 training images per person, respectively. When combining one preprocessing method with the TT method, our NS-based preprocessing method achieves the best FR accuracy of $96.29 \%, 97.45 \%$, and $98.31 \%$ using 4, 5, and 6 training images, respectively. When individually combining PP, HE, and LN with our NSbased preprocessing method, the combined preprocessing method achieves better FR accuracy than their corresponding preprocessing counterparts (PP, HE, and LN). Specifically, LN followed by the NS-based preprocessing achieves the best FR accuracy of $97.33 \%, 98.40 \%$, and $98.87 \%$ using 4,5 , and 6 training images, respectively. Correspondingly, it improves the TT's FR accuracy (the 4th row) by $3.05 \%, 2.55 \%$, and $1.28 \%$. It clearly shows the our NS-based preprocessing is the best one.

\subsection{Recognition results on FEI database}

The FEI face database has 14 images $(640 \times 480$ pixels $)$ for each of 200 individuals. They are color images with a white 
Table 1. Comparison of FR accuracy on ORL database.

\begin{tabular}{|l|c|c|c|}
\hline \multirow{2}{*}{ Compared methods } & \multicolumn{3}{|c|}{ Number of training samples } \\
\cline { 2 - 4 } & $\mathbf{4}$ & $\mathbf{5}$ & $\mathbf{6}$ \\
\hline KFDA & $93.83 \%$ & $95.50 \%$ & $96.01 \%$ \\
NeutroKFDA & $94.70 \%$ & $96.35 \%$ & $96.56 \%$ \\
Neutro+LTP+KFDA & $96.29 \%$ & $97.45 \%$ & $98.31 \%$ \\
TT(PP+LTP+KFDA) & $94.45 \%$ & $95.95 \%$ & $97.62 \%$ \\
HE+LTP+KFDA & $94.83 \%$ & $96.65 \%$ & $97.75 \%$ \\
LN+LTP+KFDA & $94.87 \%$ & $96.50 \%$ & $97.56 \%$ \\
PP+Neturo+LTP+KFDA & $95.70 \%$ & $96.80 \%$ & $97.87 \%$ \\
HE+Neutro+LTP+KFDA & $96.79 \%$ & $98.00 \%$ & $98.68 \%$ \\
LN+Neutro+LTP+KFDA & $97.33 \%$ & $98.40 \%$ & $98.87 \%$ \\
\hline
\end{tabular}

Table 2. Comparison of FR accuracy on FEI database.

\begin{tabular}{|l|c|c|c|}
\hline \multirow{2}{*}{ Compared methods } & \multicolumn{3}{|c|}{ Number of training samples } \\
\cline { 2 - 4 } & $\mathbf{4}$ & $\mathbf{5}$ & $\mathbf{6}$ \\
\hline KFDA & $81.95 \%$ & $86.00 \%$ & $87.18 \%$ \\
NeutroKFDA & $87.05 \%$ & $91.16 \%$ & $93.18 \%$ \\
Neutro+LTP+KFDA & $90.60 \%$ & $93.33 \%$ & $95.00 \%$ \\
TT(PP+LTP+KFDA) & $90.55 \%$ & $91.15 \%$ & $94.50 \%$ \\
HE+LTP+KFDA & $88.50 \%$ & $91.22 \%$ & $93.50 \%$ \\
LN+LTP+KFDA & $88.50 \%$ & $91.16 \%$ & $93.56 \%$ \\
PP+Neturo+LTP+KFDA & $90.55 \%$ & $93.16 \%$ & $95.18 \%$ \\
HE+Neutro+LTP+KFDA & $91.25 \%$ & $93.77 \%$ & $95.43 \%$ \\
LN+Neutro+LTP+KFDA & $91.70 \%$ & $95.27 \%$ & $95.75 \%$ \\
\hline
\end{tabular}

homogenous background in an upright frontal position with profile rotation of up to about 180 degrees. Subjects are between 19 and 40 years old with distinct appearance, hairstyle, and adorns [9]. Fig. 2(b) shows a sample individual from this database at the top row and the corresponding preprocessed images at the bottom row.

To speed up the processing time, we convert all FEI face images to grayscale images and normalize them to the size of $32 \times 24$ pixels. We use a subset of this database that contains the first 40 individuals for our experiments. Table 2 shows the average FR rates obtained by combining different preprocessing methods and the discriminant method (e.g., KFDA or TT method) using a different number of training images. This table clearly shows that our NS-based preprocessing followed by KFDA improves KFDA by $6.22 \%$, $6.0 \%$, and $6.88 \%$ in terms of FR accuracy using 4,5 , and 6 training images per person, respectively. When combining one preprocessing method with the TT method, our NSbased preprocessing method achieves the best FR accuracy of $90.60 \%, 93.33 \%$, and $95.00 \%$ using 4, 5, and 6 training images, respectively. When individually combining PP, HE, and LN with our NS-based preprocessing method, the combined preprocessing method achieves better FR accuracy than their corresponding preprocessing counterparts (PP, HE, and LN). Specifically, LN followed by the NS-based preprocessing achieves the best FR accuracy of $91.70 \%, 95.27 \%$, and $95.75 \%$ using 4,5 , and 6 training images, respectively. Correspondingly, it improves the TT's FR accuracy (the 4th row) by $1.27 \%, 4.52 \%$, and $1.32 \%$. It clearly shows the our NSbased preprocessing is the best one.

\section{CONCLUSIONS AND FUTURE WORK}

We propose an effective NS-based preprocessing method to remove noise and enhance facial features in face images. We then individually apply two discriminant methods, KFDA and TT, on preprocessed face images to achieve a better FR rate. Our experiments on two databases demonstrate the effectiveness of this NS-based preprocessing method to boost the performance of discriminative methods for the FR task. They also show the NS-based preprocessing can be integrated with other preprocessing methods to further improve FR accuracy.

Our major contributions are: 1) Use entropy to find the best $\alpha, \beta$, and $\gamma$ values for all database images. 2) Find the relationship among $\alpha, \beta$, and $\gamma$ which is suitable for most face images. 3) Estimate the optimal $\alpha, \beta$, and $\gamma$ values for each face image. 4) Preprocess each face image using the optimal $\alpha, \beta$, and $\gamma$ values by applying the entropy-based convergence condition. 5) Combine the NS-based preprocessing with KFDA or TT to achieve better performance.

In the future, we will use clustering and statistical techniques to find a better relationship among $\alpha, \beta$, and $\gamma$. We will also explore the possibility to employ NS-based preprocessing in color images and other applications.

\section{REFERENCES}

[1] A. F. Abate, M. Nappi, D. Riccio, and G. Sabatino, "2d and 3d face recognition: A survey," Pattern Recognition Lett., vol. 28, pp. 1885-1906, 2007.

[2] C. Wang, B. Yin, X. Bai, and Y. Sun, "Color face recognition based on 2dpca," IEEE Proc. of ICPR, pp. 1-4, 2008.

[3] R. C. Gonzalez and R. E. Woods, "Digital image processing," Prentice Hall, 2007.

[4] X. Tan and B. Triggs, "Enhanced local texture feature sets for face recognition under difficult lighting conditions," IEEE Trans. Image Proc., vol. 19, no. 6, pp. 1635-1650, 2010.

[5] H. Han, S. Shan, X. Chen, and W. Gao, "A comparative study on illumination preprocessing in face recognition," Pattern Recognition, vol. 46, pp. 1691-1699, 2013.

[6] M. Zhang, L. Zhang, and H. D. Cheng, "A neutrosophic approach to image segmentation based on watershed method," Signal Proc., vol. 90, pp. 1510-1517, 2010.

[7] A. Sengur and Y. Guo, "Color texture image segmentation based on neutrosophic set and wavelet transformation," Computer Vision and Image Understanding, vol. 115, pp. 1134-1144, 2011.

[8] F. Smarandache, "A unifying field in logics neutrosophic logic. neutrosophy, neutrosophic set, neutrosophic probability," American Research Press, 3th edition, 2003.

[9] C. E. Thomaz and G. A. Giraldi, "A new ranking method for principal components analysis and its application to face image analysis," Image and Vision Comp., vol. 28, no. 6, pp. 902-913, 2010. 